Artículo

\title{
Efecto de la exclusión de un área natural protegida en la composición y estructura arbórea
}

\author{
Enrique Buendía-Rodríguez ${ }^{18}$ \\ Eduardo Alanís-Rodríguez ${ }^{2}$ \\ Oscar A. Aguirre-Calderón ${ }^{2}$ \\ Eduardo J. Treviño-Garza ${ }^{2}$ \\ Eulogio Flores-Ayala ${ }^{1}$ \\ Fernando Carrillo-Anzures ${ }^{1}$
}

${ }^{1}$ Campo Experimental Valle de México-INIFAP. Carretera los Reyes-Texcoco km 13.5, Coatlinchán, Texcoco, Estado de México, México. AP. 10. CP. 56250. ${ }^{2}$ Facultad de Ciencias Forestales-Universidad Autónoma de Nuevo León. Carretera Linares-Cd. Victoria km 145, Linares, Nuevo León, México. CP. 67700. AP 41.

${ }^{\S}$ Autor de correspondencia: buendia.enrique@inifap.gob.mx.

\section{Resumen}

El Parque Nacional Izta-Popo es un área natural protegida (ANP) que fue creada con la finalidad de mantener la representatividad de los ecosistemas. Actualmente, no se sabe la situación de zonas que fueron excluidas de aprovechamiento forestal. El objetivo fue evaluar el efecto de la exclusión de un ANP en la composición y estructura del arbolado por medio de caracterización dasométrica de un bosque templado en la región central de México. Se seleccionaron tres sitios cuadrados de 100 x 100 m (1 ha): dos dentro del ANP (Pinus hartwegii "BPiHa" у Pinus montezumae "BPiMo") y un bajo aprovechamiento forestal (Abies religiosa "BAbRe"), donde se midió el diámetro normal $\left(\mathrm{d}_{1.30}\right)$, altura total $(\mathrm{h})$, para calcular el área basal $(\mathrm{G})$, volumen total (vt), diámetro promedio (d), altura promedio (h), índice de esbeltez (h/d), densidad (dn). El BPiMo tiene una densidad de 149 individuos, área basal $\left(39.21 \mathrm{~m}^{2} \mathrm{ha}^{-1}\right)$, volumen total $(941.82$ $\left.\mathrm{m}^{3} \mathrm{ha}^{-1}\right)$, diámetros promedios $(55.34 \mathrm{~cm})$ y altura promedio $(34.15 \mathrm{~m})$. El BPiHa tiene una densidad de 133 individuos, área basal $\left(23.27 \mathrm{~m}^{2} \mathrm{ha}^{-1}\right)$, volumen total $\left(328.47 \mathrm{~m}^{3} \mathrm{ha}^{-1}\right)$, altura promedio $(21.44 \mathrm{~m})$ y diámetros promedios $(43.83 \mathrm{~cm})$. La BAbRe, tiene una alta densidad de 315 individuos, área basal $\left(32.37 \mathrm{~m}^{2} \mathrm{ha}^{-1}\right)$, volumen total $\left(468.58 \mathrm{~m}^{3} \mathrm{ha}^{-1}\right)$, diámetros promedios $(31.71 \mathrm{~cm})$ y altura promedio $(24.22 \mathrm{~m})$. Los bosques dentro de las ANP (BPiMo y BPiHa) requieren de aprovechamiento maderable, con la finalidad de regenerar la masa forestal. El BAbRe se encuentra bajo manejo forestal y cumple con esa condición.

Palabras clave: análisis estructural, bosque templado, manejo forestal.

Recibido: febrero de 2018

Aceptado: julio de 2018 


\section{Introducción}

El Parque Nacional Iztaccíhuatl-Popocatépetl-Zoquiapán”, está ubicado en la región central de México. El Parque Nacional Izta-Popo es una de las áreas naturales protegidas (ANP) más antiguas, fue creado en 1935 con el fin de proteger las montañas que conforman la Sierra Nevada. Esta ANP fue creada con la finalidad de mantener la representatividad de los ecosistemas y su biodiversidad, asegurando la provisión de servicios ambientales mediante su conservación y manejo sustentable.

Actualmente, no se sabe cuál es el escenario de esas zonas que fueron excluidas del manejo forestal para pertenecer a ANP, o si ha sido exitoso ese tipo de manejo (no tocar) con el supuesto de una mejor conservación de los recursos bióticos. Un bosque bajo manejo genera una masa arbolada con mejores características dasométricas mejorando los servicios que esta presenta y manteniendo un flujo constante de recursos bióticos a diferencia de un bosque que se mantienen sin aprovechamiento maderable que tiende a estabilizase en el crecimiento de la masa y se mantienen estable. Una de las formas más eficientes de evaluar una masa arbolada sea cual sea se condición es a través de una caracterización dasométrica.

La caracterización dasométrica es una referencia fundamental de los patrones y las relaciones dentro de un sistema ecológico, la cual involucra regeneración, mortalidad de árboles y una variedad de interacciones entre individuos, que a su vez afecta las condiciones microclimáticas (Joao y Carvalho, 2011; Gadow et al., 2012) y es una manera de estimar la condición de los ecosistemas en un momento determinado y su evolución a través del tiempo (Gadow et al., 1998; Vargas-Larreta et al., 2010; Petritan et al., 2012; Hui y Pommerening, 2014).

Una correcta caracterización de ecosistemas, sirve para saber el estado actual de la vegetación que a su vez se usará como base para realizar una adecuada toma de decisiones para el manejo de los recursos forestales, tanto en localidades bajo aprovechamiento como en áreas naturales protegidas (Jiménez et al., 2001; Aguirre 2003b; Wehenkel et al., 2011; Bannister y Donoso, 2013). Para este propósito, se utilizan índices estructurales y variables dasométricas, que incluyen diámetro, altura, área basal, etc., con la finalidad de lograr una mejor descripción (Aguirre et al., 2003a).

A nivel mundial existe suficiente literatura para evaluar la estructura de ecosistemas forestales (Liira et al., 2007; Joao y Carvalho, 2011; Brito-Rozas y Flores-Toro, 2014). En el norte de México, existen estudios que evalúan la estructura de ecosistemas forestales, donde describen las comunidades vegetales bajo aprovechamiento forestal (Aguirre et al., 2003a; Corral et al., 2005; Solís et al., 2006; Torres et al., 2006; García-De La Cruz et al., 2013; Hernández-Salas et al., 2013) y regeneradas posperturbación (Alanís-Rodríguez et al., 2008; Ávila-Flores et al., 2012); sin embargo, en el centro de México existe escasa información de áreas conservadas bajo el esquema de área natural protegida. El presente trabajo se enfoca en comparar tres áreas forestales, dos ubicadas dentro de una área natural protegida (una de ellas con acceso restringido al público en general, otra con acceso al público) y la última, un área bajo aprovechamiento forestal, dicha comparación se realizará mediante procedimientos cuantitativos que permitirán analizar con diferente precisión la condición de las masas forestales, en cuanto a su desarrollo y así poder definir las bases para realizar una adecuada toma de decisiones para la implementación de un adecuado manejo forestal de las áreas estudiadas, como un factor indispensable para la generación de recursos tangibles e intangibles en beneficio de la región más densamente poblada de México. 
La hipótesis planteada fue que el bosque excluido tendrá un mejor estado de conservación que los bosques bajo manejo, ya que al no haber interacción con las actividades humanas no existe un deterioro del recurso. Por tal motivo, el objetivo de la presente investigación fue evaluar el efecto de la exclusión de un área natural protegida en la composición y estructura del elemento arbóreo por medio de caracterización dasométrica de un bosque templado en la región central de México, con la finalidad de determinar el estado actual del recurso forestal.

\section{Materiales y métodos}

Área de estudio. La zona de estudio se encuentra en la parte centro oriental del Eje Volcánico Transversal Mexicano (Figura 1). Se seleccionaron dos tipos de bosque en un área natural protegida (Pinus montezumae Lamb. y Pinus hartwegii Lindl.) y una masa forestal en un bosque bajo manejo (Abies religiosa (H. B. K.) Schl. et Cham.)), las cuales se localizan entre las coordenadas siguientes: $19^{\circ} 00^{\prime}$ y $19^{\circ} 28^{\prime}$ de latitud norte y $98^{\circ} 35^{\prime}$ y $98^{\circ} 46^{\prime}$ de longitud oeste (Datum WGS 84).

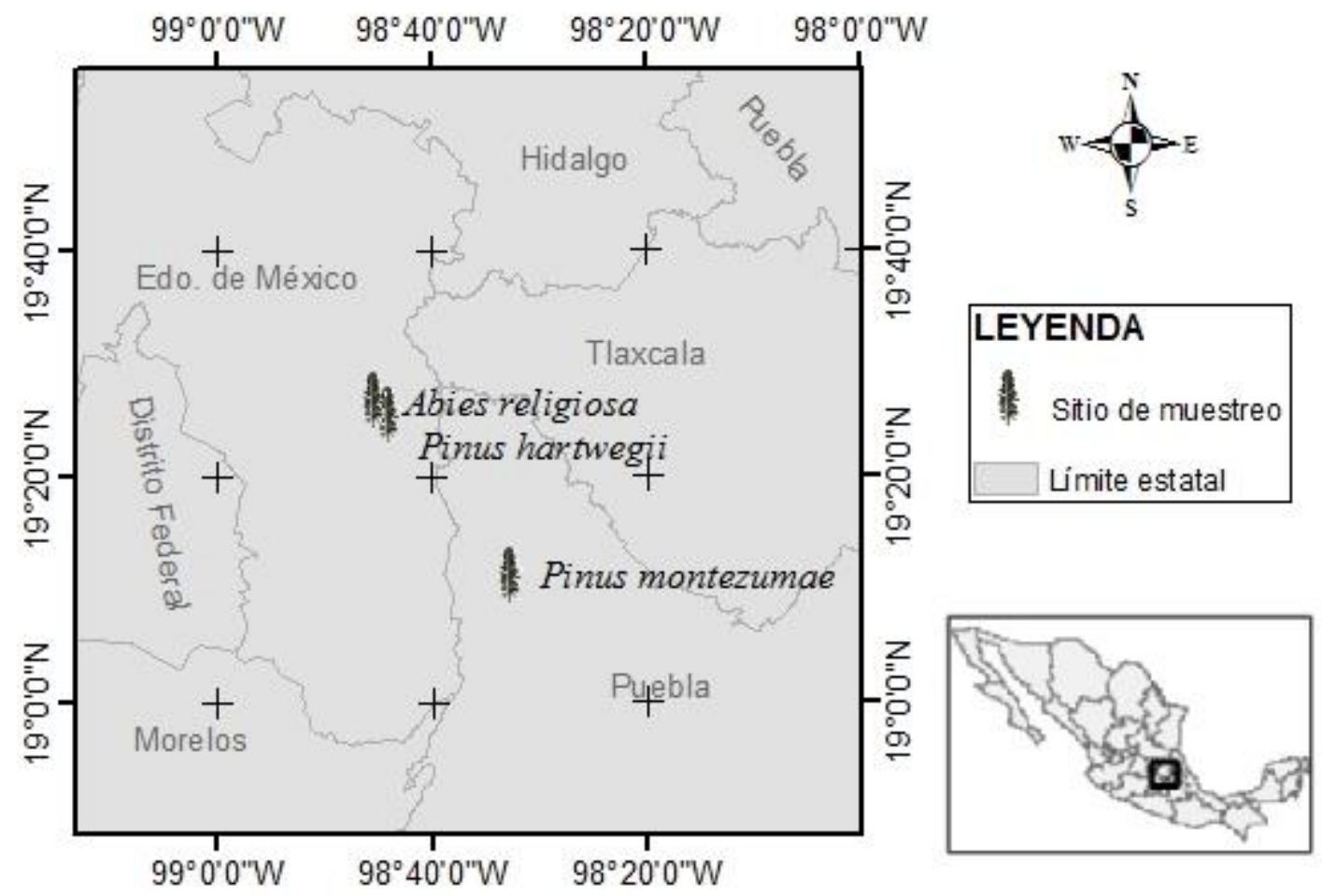

Figura 1. Mapa de localización del área de estudio.

Para satisfacer el objetivo planteado, se seleccionaron tres parcelas de muestreo con características geográficas similares (Cuadro 1). La masa de Abies religiosa ha sido aprovechada madereramente mediante el Método Mexicano de Ordenación de Bosques Irregulares (MMOBI), después de que se levantó la veda en el Estado de México (1969), la de Pinus hartwegii no se ha aprovechado madereramente desde 1948 que se determinó como parte del parque nacional Izta-Popo (DOF, 1948), y la masa de Pinus montezumae es un caso especial ya que desde 1963 se encuentra ubicado en lo que fue el Campo experimental forestal "San Juan Tetla" del Instituto Nacional de 
Investigaciones Forestal, Agrícola y Pecuaria (INIFAP) y solo se hicieron algunas cortas de saneamiento, anterior de esa fecha (de 1948 a 1963) fue aprovechado por la Unidad Industrial de Explotación Forestal que abasteció de materia prima a la fábrica de papel de San Rafael.

Cuadro 1. Características geográficas y datos climáticos de los tres sitios de muestreo.

\begin{tabular}{|c|c|c|c|}
\hline Vegetación & $\begin{array}{c}\text { Bosque de Pinus } \\
\text { montezumae (BPiMo) }\end{array}$ & $\begin{array}{l}\text { Bosque de Pinus } \\
\text { hartwegii (BPiHa) }\end{array}$ & $\begin{array}{l}\text { Bosque de Abies } \\
\text { religiosa (BAbRe) }\end{array}$ \\
\hline Coordenadas (UTM) & $547311 \mathrm{~W} 2121047 \mathrm{~N}$ & $27518 \mathrm{~W} 2147240 \mathrm{~N}$ & $525049 \mathrm{~W} 2149315 \mathrm{~N}$ \\
\hline Altitud (msnm) & 3300 & 3600 & 3000 \\
\hline Clima & $\mathrm{C}(\mathrm{w} 2)(\mathrm{w})\left(\mathrm{b}^{\prime}\right)\left(\mathrm{I}^{\prime}\right) \mathrm{g}$ & $\mathrm{C}(\mathrm{w} 0)(\mathrm{w}) \mathrm{b}\left(\mathrm{I}^{\prime}\right) \mathrm{g}$ & $\mathrm{C}(\mathrm{w} 0)(\mathrm{w}) \mathrm{b}\left(\mathrm{I}^{\prime}\right) \mathrm{g}$ \\
\hline${ }^{*}$ Temperatura media anual $\left({ }^{\circ} \mathrm{C}\right)$ & 12.5 & 15.8 & 15.8 \\
\hline Precipitación media anual (mm) & 815 & 650 & 650 \\
\hline${ }^{* *}$ Tipo de suelo & Andosol húmico & Cambisol éutrico & Cambisol éutrico \\
\hline
\end{tabular}

Toma de datos. Se evaluaron tres comunidades vegetales del bosque templado-frío (BPiMo, BPiHa y BAbRe) del oriente del Estado de México y occidente de Puebla. El diseño consistió en establecer tres sitios cuadrados de 100 x $100 \mathrm{~m}$ ( $1 \mathrm{ha}$ ), los cuales se subdividieron en nueve subsitios de 33.33 x $33.33 \mathrm{~m}\left(1110 \mathrm{~m}^{2}\right)$. En los subsitios se tomó información dendrométrica de las especies arbóreas presentes, efectuándose mediciones de diámetro normal $\left(\mathrm{d}_{1.30}\right)$, altura total $(\mathrm{h})$, de todo el arbolado $\geq 10 \mathrm{~cm}$ de diámetro normal.

Análisis de datos. Con la información dendrométrica recabada en campo se calculó para cada sitio el área basal (G), volumen total (vt), diámetro promedio (d), altura promedio (h), índice de esbeltez (h/d), densidad (dn).

El área basal por unidad de superficie (1 ha), se definió como la suma de las secciones trasversales de todos los fustes a nivel del diámetro normal $\left(\mathrm{d}_{1.30}\right)$. Para los valores por hectárea del volumen total, se realizó la sumatoria de valores individuales, los cuales se calcularon con las ecuaciones alométricas que se enlistan en el Cuadro 2.

Cuadro 2. Ecuaciones usadas para calcular el volumen total por árbol.

\begin{tabular}{ccc}
\hline Especie & Fórmula & Autor \\
\hline Pinus montezumae & $\mathrm{v}=$ Anti $\ln \left(-11.285+1.1183 * \ln \left(\mathrm{d}^{2} * \mathrm{~h}\right)\right)$ & Acosta y Carrillo (2008) \\
Pinus hartwegii & $\mathrm{v}=$ Anti $\ln (-9.5377+1.9649 * \ln \mathrm{d}+0.89055 * \ln \mathrm{h})$ & Rodríguez y Padilla (1976) \\
Abies religiosa & $\mathrm{v}=0.00004432 * \mathrm{~d}^{2.06319} * \mathrm{~h}^{0.86404}$ & PROBOSQUE (1990) \\
Quercus laurina & $\mathrm{v}=0.000066 * \mathrm{~d}^{2.138} * \mathrm{~h}^{0.6899}$ & Pacheco (2011) \\
$*^{*}$ Quercus rugosa & $\mathrm{v}=0.000066 * \mathrm{~d}^{2.138} * \mathrm{~h}^{0.6899}$ & Pacheco (2011) \\
Alnus jorullensis & $\mathrm{v}=0.000016737 * \mathrm{~d}^{2} * \mathrm{~h}+0.000526 \mathrm{~d}^{2}$ & Quintana (1999) \\
Cupressus lindleyi & $\mathrm{v}=0.00007204 * \mathrm{~d}^{1.74008} * \mathrm{~h}^{1.04811}$ & PROBOSQUE (1990) \\
Otras latifoliadas & $\mathrm{v}=0.00009001 * \mathrm{~d}^{2.38434} * \mathrm{~h}^{0.16699}$ & PROBOSQUE (1990) \\
\hline
\end{tabular}

$\mathrm{v}=\mathrm{es}$ volumen total; $\mathrm{d}=$ es el diámetro normal; $\mathrm{y} \mathrm{h}=\mathrm{es}$ la altura; ${ }^{* *}=$ se calculó con la misma fórmula que el Q. laurina, ya que presentan similares características morfológicas. 
Para calcular por hectárea del índice de esbeltez se obtuvo un promedio de la relación (h/d), la densidad de una población (dn) fue el número de individuos.

Para detectar si existen diferencias significativas de las variables estudiadas (área basal, volumen total, diámetro promedio, altura total, índice de esbeltez y densidad para los tres tipos de masas forestales estudiadas, divididas en nueve subsitios de muestreo, se aplicó un análisis de varianza de un factor (Anova). En caso de encontrar diferencias significativas $(p<0.05)$ se procedió con la comparación múltiple de medias mediante la prueba de Tukey (Zar, 2010). Los análisis fueron realizados con el paquete estadístico SPSS $^{\circledR}$ (ver. 19.0).

\section{Resultados}

Caracterización dasométrica. Dentro del área de estudio se encontraron nueve especies arbóreas, cinco de estas son coníferas y cuatro latifoliadas. De estas especies, tres son de importancia comercial maderable: Pinus hartwegii, P. montezumae y Abies religiosa. La masa forestal con mayor número de individuos por hectárea fue Abies religiosa con 315, en tanto para las variables dasométricas la mejor masa fue la de Pinus montezumae, con valores de área basal de $39.21 \mathrm{~m}^{2}$ $\mathrm{ha}^{-1}$ y un volumen de $941.82 \mathrm{~m}^{3} \mathrm{ha}^{-1}$ en una población de 149 individuos por hectárea. En cambio, los valores menores son para la masa de Pinus hartwegii donde se registraron 133 individuos por hectárea, con valores de área basal de $23.27 \mathrm{~m}^{2} \mathrm{ha}^{-1} \mathrm{y}$ un volumen de $328.47 \mathrm{~m}^{3} \mathrm{ha}^{-1}$ (Cuadro 3).

Cuadro 3. Características dasométricas: área basal, volumen total, diámetro promedio, altura promedio, índice de esbeltez y densidad de las especies de las tres áreas de muestreo.

\begin{tabular}{lcccccc}
\hline Especie & $\begin{array}{c}\text { Diámetro } \\
\text { promedio } \\
(\mathrm{cm})\end{array}$ & $\begin{array}{c}\text { Altura } \\
\text { promedio } \\
(\mathrm{m})\end{array}$ & $\begin{array}{c}\text { Área basal } \\
\left(\mathrm{m}^{2} \mathrm{ha}^{-1}\right)\end{array}$ & $\begin{array}{c}\text { Volumen } \\
\text { total } \\
\left(\mathrm{m}^{3} \mathrm{ha}^{-1}\right)\end{array}$ & $\begin{array}{c}\text { Índice de } \\
\text { esbeltez } \\
\text { promedio }^{-1}\end{array}$ & $\begin{array}{c}\text { Densidad } \\
\left(\mathrm{h} \mathrm{d}^{-1}\right)\end{array}$ \\
\hline Pinus montezumae Lamb. & 55.34 & 34.15 & 39.21 & 941.82 & 65 & 149 \\
Pinus hartwegii Lindl & 43.83 & 21.44 & 23.27 & 328.47 & 50 & 133 \\
Abies religiosa (H. B. K.) & 31.71 & 24.22 & 32.37 & 468.58 & 84 & 315 \\
Schl. Et Cham & & & & & & \\
\hline
\end{tabular}

Con respecto a los diámetros de Pinus montezumae, estos se distribuyen en forma bimodal, presentando un mayor número de individuos en las categorías diamétricas de 55, 60 y $65 \mathrm{~cm}$ (42.95\%) y menor cantidad de árboles en las categorías menores de $40 \mathrm{~cm}$ (14.1\%) (Figura 2A). Dicha masa está conformada por dos estratos en altura, y la mayoría de los individuos se encuentran en categorías de 35 y $40 \mathrm{~m}(67.11 \%)$ y un estrato inferior con individuos con alturas menores a 10 m (Figura 2B). En la gráfica de dispersión (Figura 2C), se puede distinguir que existe un amplio número de árboles en clases diamétricas de 40 a $80 \mathrm{~cm}$ y alturas de 25 a $55 \mathrm{~m}$ y un menor número se distribuye entre 15 y $35 \mathrm{~cm}$ de diámetro. En su conjunto esta masa está conformada por un rodal sobremaduro de dos estratos en altura, con árboles mayores a $40 \mathrm{~cm}$ de diámetro que podrían ser susceptibles a aprovechamiento maderero.

La masa de Pinus hartwegii tiene una distribución bimodal con un mayor número de individuos en las categorías diamétricas de 25 hasta $75 \mathrm{~cm}$ (Figura 2D), teniendo mayor frecuencia en las categorías 45, 50, 55 y $60 \mathrm{~cm}(53.39 \%)$. Para la distribución en altura se encuentra en todas las 
categorías con un mayor número de árboles en las categorías de 25 y 30 m (65.41\%) (Figura 2D). En la gráfica de dispersión (Figura $2 \mathrm{~F}$ ), se puede distinguir que existe un amplio número de árboles maduros en clases diamétricas de 45 a $55 \mathrm{~cm}$ y alturas de 20 a $30 \mathrm{~m}$. También se observa un grupo de individuos jóvenes menores a $15 \mathrm{~cm}$ de diámetro y $10 \mathrm{~m}$ de altura (Figura 2F).

Esta masa está conformada por dos estratos en altura y dominados con individuos de $25 \mathrm{~m}$ de altura en el estrato superior y un estrato inferior con árboles en la categoría de $5 \mathrm{~m}$ de altura. En la Figura 2D, se observa una distribución diamétrica con presencia en las categorías de 10 hasta $75 \mathrm{~cm}$ (excepto en la categoría de $20 \mathrm{~cm}$ ). En la categoría faltante $(20 \mathrm{~cm})$ se refleja en una menor frecuencia de individuos en la categoría de altura de $10 \mathrm{~m}$. Esta masa, está conformado por un rodal maduro de dos estratos en altura, con árboles mayores a $40 \mathrm{~cm}$ de diámetro que pueden ser susceptibles a aprovechamiento maderero.

Para el bosque de Abies religiosa los diámetros tienen una distribución Poisson con una asimetría a la izquierda, con mayor presencia en la categoría de 15, 20 y $25 \mathrm{~cm}$ (41.57\%) (Figura 2G). Esta masa está conformada por dos estratos en altura, y los individuos se encuentran con mayor frecuencia en la categoría de $15 \mathrm{~m}(31.43 \%)$ y en menor medida en la categoría $35 \mathrm{~m}(21.27 \%)$ (Figura 2H). En la gráfica de dispersión (Figura 2I), se puede distinguir que existe una distribución de árboles en todas las clases diamétricas hasta $80 \mathrm{~cm}$ y alturas de $45 \mathrm{~m}$, de una forma ascendente uniforme y un individuo aislado de mayor diámetro en la categoría de $100 \mathrm{~cm}$ y dominado por individuos con alturas de 30 - $35 \mathrm{~m}$ de altura y el segundo estrato con árboles de $15 \mathrm{~m}$ y pocos individuos mayores a $40 \mathrm{~cm}$ de diámetro que pueden ser susceptibles a aprovechamiento maderero.

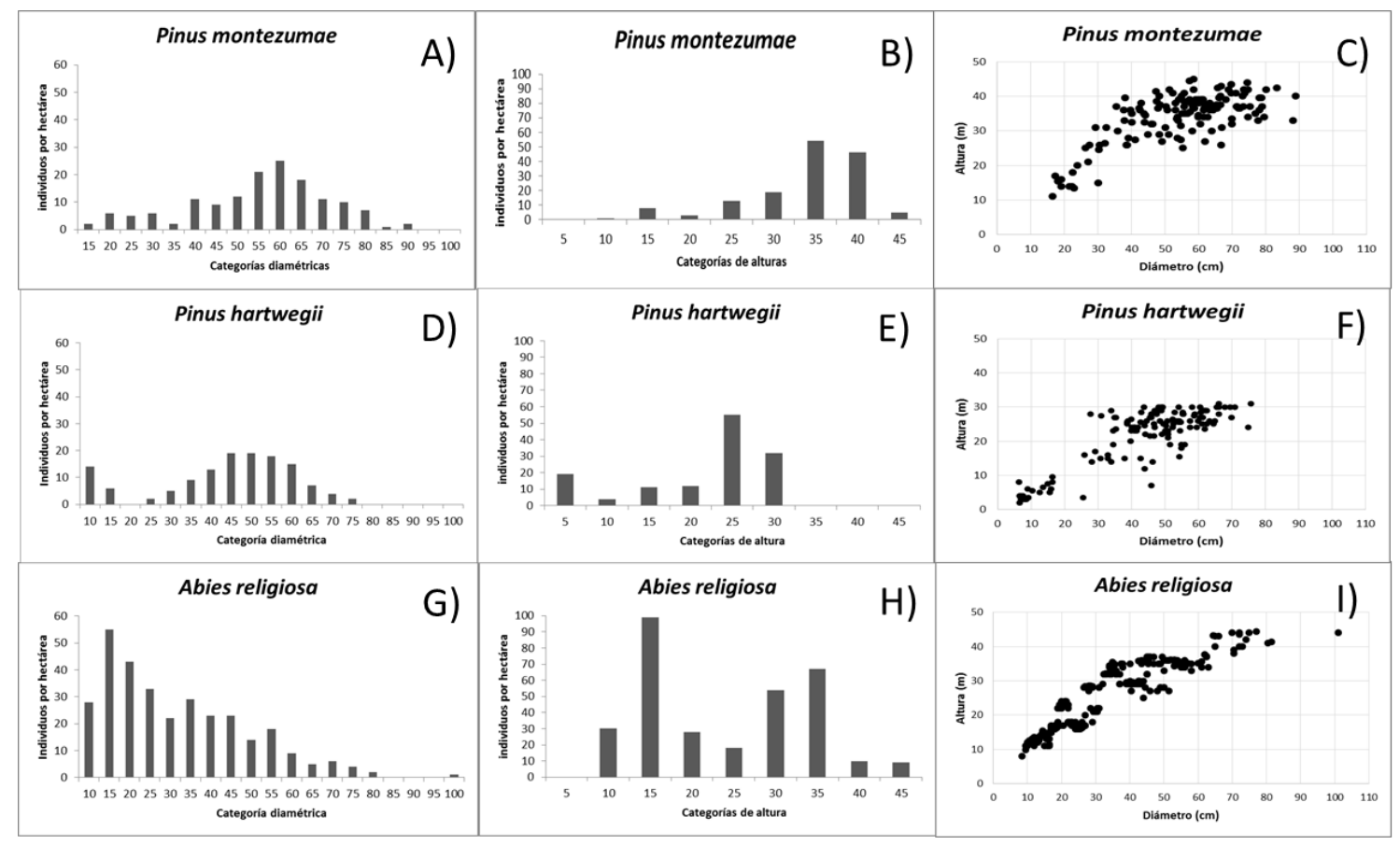

Figura 2. Distribución de individuos de las especies: A) categorías diamétricas; B) categorías de alturas; C) diagrama de dispersión (altura-diámetro) de Pinus montezumae; D) categorías diamétricas; E) categorías de alturas; F) diagrama de dispersión (altura-diámetro) de Pinus hartwegii; G) categoría de diámetro; H) categoría de altura; I) diagrama de dispersión (altura-diámetro) de Abies religiosa. 
Comparación entre tres masas forestales. El diámetro promedio presenta diferencias significativas entre las tres áreas $(p<0.05)$. El bosque de Pinus montezumae tiene el valor más alto de $55.34 \pm 2.2$ $\mathrm{cm})$, seguido del bosque de Pinus hartwegii $(43.83 \pm 3.2 \mathrm{~cm})$ y el menor, el bosque de Abies religiosa $(31.71 \pm 0.9 \mathrm{~cm})$ (Figura $3 \mathrm{~A})$.

La altura promedio presenta diferencias significativas entre las tres áreas $(p<0.05)$. El bosque de Pinus montezumae tiene el valor más alto de área basal $(34.15 \pm 2.1 \mathrm{~m})$ y el bosque de Abies religiosa (24.22 $\pm 0.9 \mathrm{~m}$ ) y el menor, el bosque de Pinus hartwegii (21.44 $\pm 2 \mathrm{~m}$ ) (Figura 3B).

El área basal presenta diferencias significativas entre las tres áreas ( $p<0.05)$. El bosque de Pinus montezumae tiene el valor más alto de área basal $\left(41.61 \pm 3.8 \mathrm{~m}^{2} \mathrm{ha}^{-1}\right)$ y el bosque de Abies religiosa $\left(34 \pm 1.63 \mathrm{~m}^{2} \mathrm{ha}^{-1}\right)$ y el menor, el bosque de Pinus hartwegii $\left(23.27 \pm 4.7 \mathrm{~m}^{2} \mathrm{ha}^{-1}\right)$ presentan similitud con los otros dos bosques (Figura 3C).

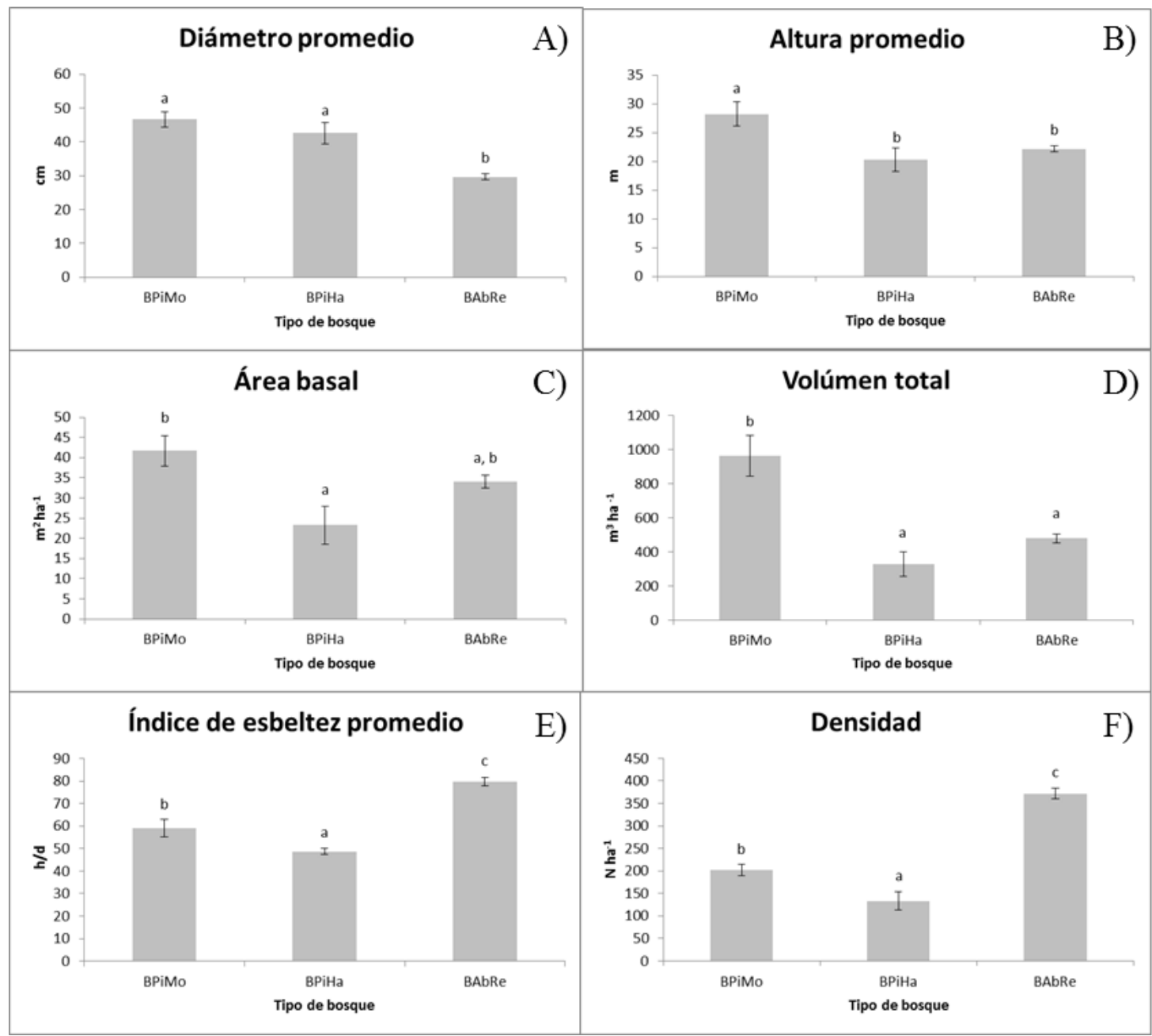

Figura 3. Medias y error estándar: A) diámetro promedio (cm); B) altura promedio (m); C) área basal $\left(\mathrm{m}^{2} \mathrm{ha}^{-1}\right)$; D) volumen total $\left(\mathrm{m}^{3} \mathrm{ha}^{-1}\right)$; E) índice de esbeltez $\left.\left(\mathrm{h} \mathrm{d}^{-1}\right) ; \mathrm{y} \mathrm{F}\right)$ densidad (árboles $\left.(\mathbf{N}) \mathbf{h a}^{-1}\right)$. BPiMo= bosque de Pinus montezumae; $\mathrm{BPiHa}=$ bosque de Pinus hartwegii; $\mathrm{BAbRe}=$ bosque de Abies religiosa. Medias seguidas de diferentes letras (a, b, c) indican diferencias significativas para $p<0.05$. 
El volumen total también presenta diferencia significativa entre parcelas de muestreo $(p<0.05)$. El Bosque de Pinus montezumae fue el que presentó el mayor nivel de volumen total (962.67 \pm 119 . $38 \mathrm{~m}^{3} \mathrm{ha}^{-1}$ ) siendo distinto a los otros dos bosques. Los bosques de Abies religiosa (478.91 \pm 27.41 $\left.\mathrm{m}^{3} \mathrm{ha}^{-1}\right)$ y el bosque de Pinus hartwegii $\left(328.47 \pm 72.75 \mathrm{~m}^{3} \mathrm{ha}^{-1}\right.$ ) no presentan diferencias significativas (Figura 3D).

Con respecto al índice de esbeltez difieren los tres tipos de bosques $(p<0.05)$. El bosque de Abies religiosa presenta los índices de esbeltez más altos $(68.5 \pm 1.86)$, seguido del bosque de Pinus montezumae (59 \pm 3.91$)$, y con menor índice el bosque de Pinus hartwegii $(50 \pm 1.34)$ (Figura 3E).

Por último, la densidad también tiene diferencia significativa entre tipos de bosques $(p<0.05)$. El de mayor densidad es el bosque de Abies religiosa ( $369 \pm 12.64$ árboles ha ${ }^{-1}$ ), seguido del bosque de Pinus montezumae (202 \pm 12.56 árboles ha $^{-1}$ ), y al final el bosque de Pinus hartwegii (133 \pm 20.11 árboles ha-1) (Figura 3F).

\section{Discusión}

La masa de Pinus montezumae tiene una densidad de 149 individuos y cuenta con valores altos en área basal $\left(39.21 \mathrm{~m}^{2} \mathrm{ha}^{-1}\right)$, y volumen total $\left(941.82 \mathrm{~m}^{3} \mathrm{ha}^{-1}\right)$; lo anterior, se explica por lo diámetros promedios de $55.34 \mathrm{~cm}$ y altura promedio de $34.15 \mathrm{~m}$, lo que refleja individuos con bajo índice de esbeltez (65) encontrándose arboles robustos. Estos valores son resultado de no ser aprovechados en más de 50 años.

En la masa de bosque Pinus hartwegii tiene una densidad de 133 individuos y cuenta con valores bajos en área basal $\left(23.27 \mathrm{~m}^{2} \mathrm{ha}^{-1}\right) \mathrm{y}$ volumen total $\left(328.47 \mathrm{~m}^{3} \mathrm{ha}^{-1}\right)$, estos valores son el resultado de individuos con valores de índice de esbeltez (50), lo que refleja árboles de poca altura promedio de $21.44 \mathrm{~m}$ y diámetros promedios de $43.83 \mathrm{~cm}$. Lo anterior, se explica por la altitud (3 $600 \mathrm{msnm})$ de la masa en el área de estudio, por lo cual están sujetos a un mayor riesgo de derribo o ruptura del fuste por viento.

A diferencia de las masas de BAbRe, tiene una alta densidad de Abies religiosa (315 árboles ha ${ }^{-1}$ ), que es a especie dominante y cuenta con valores altos en área basal $\left(32.37 \mathrm{~m}^{2} \mathrm{ha}^{-1}\right)$ y volumen total $\left(468.58 \mathrm{~m}^{3} \mathrm{ha}^{-1}\right)$; lo anterior, se explica por lo diámetros promedios de $31.71 \mathrm{~cm}$ y altura promedio de $24.22 \mathrm{~m}$, lo que refleja individuos con altos valores del índice de esbeltez (84) resultado de a su elevada densidad, este fenómeno es debido principalmente a que el Abies religiosa es tolerante a la sombra.

La masa bajo manejo forestal (BAbRe) tienen mayor densidad en categorías diamétricas pequeñas manteniendo la dinámica del bosque, ya que se genera nuevos individuos para las categorías diamétricas subsecuentes, a diferencia de los bosques maduros o sobremaduros (BPiHa y BPiMo, respectivamente) que tienen mayor representatividad en categorías diamétricas grandes teniendo mayor número de árboles sobremaduros, que son susceptibles a plagas y enfermedades. 
La masa de BPiMo presentó valores altos de área basal $\left(41.61 \mathrm{~m}^{2} \mathrm{ha}^{-1}\right)$, volumen total $\left(962.67 \mathrm{~m}^{3}\right.$ $\mathrm{ha}^{-1}$ ), densidad (202 árboles ha ${ }^{-1}$ ) e índice de esbeltez (65). Estos valores son el resultado del manejo de conservación (no extracción de madera), ya que se encuentra dentro del área del Campo Experimental "San Juan Tetla" del INIFAP, la cual está totalmente protegida del clandestinaje. En otros estudios como los realizados por Zepeda y Acosta (2000), en un área similar, dentro del mismo campo experimental, pero en una zona con acceso a los pobladores de la región, los resultados presentan valores menores los cuales fueron de $25.12 \mathrm{~m}^{2} \mathrm{ha}^{-1}$ y $28.76 \mathrm{~m}^{2} \mathrm{ha}^{-1}$ (área basal), $341.93 \mathrm{~m}^{3} \mathrm{ha}^{-1}$ y $469.6 \mathrm{~m}^{3} \mathrm{ha}^{-1}$ (volumen total) y 182 árboles ha ${ }^{-1}$ y 168 árboles ha ${ }^{-1}$ (densidad). Con respecto al índice de esbeltez (65), son valores altos comparados con estudios de áreas bajo manejo como los reportados por Corral-Rivas et al. (2005) que fueron de 44 y 57 y los de 48 (Aguirre et al., 2003a), debido principalmente a corta de arbolado de mayor diámetro, dejando diámetros pequeños con buenas alturas que se espera que incremente en diámetro en el transcurso del ciclo de corta.

Para la masa de BPiHa registró los menores indicadores en todas las características estudiadas. Ya que es una masa que se encuentra en la zona del Parque Nacional Izta-Popo, pero son zonas como poca seguridad y son objeto de clandestinaje por parte de los pobladores de la región. En este estudio se encontraron valores menores de área basal $\left(23.29 \mathrm{~m}^{2} \mathrm{ha}^{-1}\right)$, volumen total $\left(328.47 \mathrm{~m}^{3} \mathrm{ha}^{-}\right.$ ${ }^{1}$ ), densidad (133 árboles ha ${ }^{-1}$ ) y índice de esbeltez (50), a los encontrado en bosques bajo manejo como los reportados por García (2000) que fueron de $25.75 \mathrm{~m}^{2} \mathrm{ha}^{-1} \mathrm{y}$ los reportados por Aguirre $e t$ al. (2003a) 35.9 - $48.2 \mathrm{~m}^{2} \mathrm{ha}^{-1}$, con un valor de índice de esbeltez que van desde 43 hasta 64 para diferentes rodales. Para una zona con condiciones climáticas similares, pero en áreas bajo manejo forestal (MMOBI) PROBOSQUE (2010) reporta un área basal de $35 \mathrm{~m}^{2} \mathrm{ha}^{-1} \mathrm{y}$ un volumen $965 \mathrm{~m}^{3}$ $\mathrm{ha}^{-1}$ total de para el grupo de pino 2 (agrupación generada por PROBOSQUE con Pinus leiophylla, $P$. hartwegii y $P$. rudis) el municipio de Texcoco.

La masa de BAbRe fue el que presentó los valores más altos en el índice de esbeltez y densidad. Estos valores son el resultado del manejo forestal que se lleva a cabo en esta zona (MMOBI), la cual facilita la regeneración natural manteniendo un número alto de los individuos de categorías diamétricas menores. Los valores encontrados en este estudio para BAbRe de área basal $\left(34 \mathrm{~m}^{2} \mathrm{ha}^{-1}\right)$, volumen total $\left(468.58 \mathrm{~m}^{3} \mathrm{ha}^{-1}\right)$, índice de esbeltez (84) y densidad (369 árboles $\mathrm{ha}^{-1}$ ) fueron menores a los reportados diversos autores para bosques bajo manejo como los realizados por PROBOSQUE (2010) para área basal $\left(50 \mathrm{~m}^{2} \mathrm{ha}^{-1}\right)$ y similares en volumen $\left(417 \mathrm{~m}^{3} \mathrm{ha}^{-1}\right)$ valores promedio registrados en condiciones parecidos de manejo para el municipio de Texcoco. Con respecto a los valores reportados por Aguirre et al. (2003a) de índice de esbeltez son menores (55 y 60) para Abies vejari, que a los registrados en este estudio (84). También los valores de área basal fueron menores a reportados por Cuevas-Guzmán et al. (2011) (48.5 a $60.5 \mathrm{~m}^{2} \mathrm{ha}^{-1}$ ) y densidades que variaron de 608 a 954 árboles ha ${ }^{-1}$. En tanto que los valores de área basal fueron mayores a los reportados por Encina-Domínguez et al. (2008) $\left(16.44 \mathrm{~m}^{2} \mathrm{ha}^{-1}\right)$ con una densidad de 439 árboles ha-1 que fueron mayores a los reportados en este estudio.

La diversidad y estructura de los bosques comparados revelan que en rodales con condiciones similares existe una diferencia en composición y estructura, lo cual es un ejemplo de la complejidad ecológica que ocasiona la exclusión de un área forestal. 


\section{Conclusiones}

Los resultados indican que si existe un efecto de la exclusión de un área natural protegida en la composición y estructura del elemento arbóreo. Por tal motivo, se rechaza la hipótesis de que las zonas excluidas del manejo forestal se encuentran en un mejor estado de conservación.

Las masas de BPiHa y BPiMo que se encuentran dentro del ANP, presentan bosques irregulares (bosques maduros y sobremaduros), con similar comportamiento, ya que en el BPiHa no se ha aplicado ningún tipo de manejo desde 1948 ya que se encuentra a una altitud mayor a los 3600 msnm y el BPiMo se encuentra dentro de las instalaciones del Campo Experimental "San Juan Tetla" del INIFAP. En cambio, la masa BAbRe que se encuentra fuera del área de natural protegida y presenta manejo forestal tiene comportamiento de un bosque regular, debido al aprovechamiento mediante el MMODI, propiciando bosques con individuos jóvenes.

Los bosques que se encuentran dentro de las áreas naturales protegidas (BPiMo y BPiHa) requieren de aprovechamiento maderable, con la finalidad de regenerar la masa y tener individuos jóvenes que pueda brindar todos los servicios tangibles e intangibles. Ya que al tener individuos de diferente estructura propiciara una mayor cantidad de microclimas lo que se reflejara en mayor microhábitats, que facilitaran el establecimiento de nueva vida silvestre. En tanto que el BAbRe, que se encuentra bajo manejo forestal ya cumple con esa condición.

La caracterización dasométrica de este tipo de bosques brinda un panorama detallado del estado de las masas forestales, lo cual soporta acciones para el manejo forestal sustentable. Las variables estimadas en la presente investigación evalúan atributos básicos y sensibles de las comunidades vegetales a distintas presiones naturales y antropogénicas.

\section{Literatura citada}

Acosta, M. M. y Carrillo, A. F. 2008. Tabla de volumen total con y sin corteza para Pinus montezumae Lamb. En el estado de Hidalgo. Instituto Nacional de Investigaciones Forestales, Agrícolas y Pecuarias (INIFAP)-Campo Experimental Pachuca. Pachuca, Hidalgo. Folleto técnico núm. 7. 20 p.

Aguirre, O.; Jiménez, J.; Kramer, H. y Akça, A. 2003a. Análisis estructural de ecosistemas forestales en el Cerro del Potosí, Nuevo León, México. Ciencia UANL. 6(2):219-225.

Aguirre, O.; Hui, G.; Gadow, K. and Jiménez, J. 2003b. An analysis of spatial forest structure using neighbourhood- based variables. Forest Ecol. Manag. 183:137-145.

Alanís-Rodríguez, E.; Jiménez-Pérez, J.; Espinoza-Vizcarra, D.; Jurado-Ybarra, E.; AguirreCalderón, O. y González-Tagle, M. 2008. Evaluación del estrato arbóreo en un área restaurada post-incendio en el parque ecológico Chipinque, México. Rev. Chapingo Ser. Cienc. Forest. Amb. 14(2):113-118.

Ávila-Flores, D. Y.; González-Tagle, M. A.; Jiménez-Pérez, J.; Aguirre-Calderón, O. A.; TreviñoGarza, E. J. y Vargas-Larreta, B. 2012. Estructura de rodales Pinus hartwegii afectados por incendios utilizando parámetros de vecindad en la sierra madre oriental, México. Trop. Subtrop. Agroecosys. 15:377-387. 
Bannister, J. R. and Donoso, P. J. 2013. Forest typification to characterize the structure and composition of old-growth evergreen forests on Chiloe Island, North Patagonia (Chile) Forests. 4(4):1087-1105; doi:10.3390/f4041087.

Brito-Rozas, E. y Flores-Toro, L. 2014. Estructura y dinámica de los bosques de belloto el norte (Beilschmiedia miersii) de la Cordillera El Melón, comuna de Nogales, región de Valparaíso, Chile. Rev. Bosque. 35(1):13-21. DOI: 10.4067/S0717-92002014000100002.

Corral, R. J. J.; Aguirre, C. O. A.; Jiménez, P. J. y Corral, R. S. 2005. Un análisis del efecto del aprovechamiento forestal sobre la diversidad estructural en el bosque mesófilo de montaña "El cielo", Tamaulipas, México. Invest. Agrar. Sist. Recur. For. 14(2):217-228.

Cuevas-Guzmán, R.; Cisneros-Lepe, E. A.; Jardel-Peláez, E. J.; Sánchez-Rodríguez, E. V.; Guzmán-Hernández, L.; Núñez-López, N. M. y Rodríguez-Guerrero, C. 2011. Análisis estructural y de diversidad en los bosques de Abies de Jalisco, México. Rev. Mex. Biod. 82:1219-1233.

DOF. 1948. Segunda sección. Decreto 1. Transitorio 1. http://www.dof.gob.mx/nota_to_imagen_ fs.php?cod_diario=187907\&pagina=7\&seccion $=2$.

Encina-Domínguez J. A.; Encina-Domínguez, F. J.; Mata-Rocha, E. y Valdés-Reyna, J. 2008. Aspectos estructurales, composición florística y caracterización ecológica del bosque de oyamel de la sierra de Zapalinamé, Coahuila, México. Bol. Soc. Bot. Méx. 83:13-24.

Gadow, K.; Hui, G. and Albert, M. 1998 Das Winkelmassein Strukturparameter zur Beschreibung der Individualverteilung in Waldbeständen. Centralblatt für das gesamte Forstwesen. 115(1):1-9.

Gadow, K. v.; Yu, Z. C.; Wehenkel, C.; Pommerening, A.; Corral-Rivas, J.; Korol, M.; Myklush, S.; Ying, H. G.; Kiviste, A. and Hai, Z. X. 2012. Forest structure and diversity. In: Pukkala, K. T.; Gadow, J v. Finland y Tomé, M. (Eds.). Continuous Cover Forestry. Managing Forest Ecosystems. Second edition. 29-83 pp.

García, G. R. 2000. Comportamiento de la dinámica sucesional de Abies religiosa (hbk) schl. Et. Cham. y pinus Hartwegii Lindl. en la Estación forestal experimental zoquiapan, estado de México. Tesis de Licenciatura. Universidad Autónoma Chapingo, División de Ciencias Forestales. Chapingo, Estado de México. 88 p.

García-De la Cruz, J.; Olivares-López, L. A. y Ramos-Prado, J. M. 2013. Estructura y composición arbórea de un fragmento de bosque mesófilo de montaña en el estado de Veracruz. Rev. Chapingo Ser. Cienc. Forestal Amb. 19(1):91-101. doi: 10.5154/r.rchscfa.2012.03.025.

García E. 1981. Modificaciones al sistema de clasificación climática de Köppen. Offset Larios, México, DF. 286 p.

Hernández-Salas J. O. A.; Aguirre-Calderón, E.; Alanís-Rodríguez, J.; Jiménez-Pérez, E. J.; Treviño-Garza, M. A.; González-Tagle, C.; Luján-Alvárez, J. M.; Olivas-García, I. A. y Domínguez-Pereda. 2013. Efecto del manejo forestal en la diversidad y composición arbórea de un bosque templado del Noreste de México. Rev. Chapingo Ser. Cienc. Forestal Amb. 19(2):189-199. doi: 10.5154/r.rchscfa.2012.08.052.

Hui, G. and Pommerening, A. 2014. Analysing tree species and size diversity patterns in multispecies uneven-aged forests of Northern China. Forest ecology and management 316:125138. http://dx.doi.org/10.1016/j.foreco.2013.07.029.

INIFAP-CONABIO. 1995. Instituto Nacional de Investigaciones Forestales y AgropecuariasComisión Nacional para el Conocimiento y Uso de la Biodiversidad. Mapa de edafología. Escalas 1:250 000 y 1:1 000 000. México, DF. 
Joao, P. and Carvalho, F. 2011. Composition and structure of natural mixed-oak stands in northern and central Portugal. Forest Ecology and Management. 262:1928-1937. doi:10.1016/j.foreco.2011.04.020.

Jiménez, P.; Aguirre, C. O. J. y Kramer, H. 2001. Análisis de la estructura horizontal y vertical en un ecosistema multicohortal de pino-encino en el norte de México. Invest. Agr. Sist. Recur. For. 10(2):355-366.

Liira, J.; Sepp, T. and Parrest, O. 2007. The forest structure and ecosystem quality in conditions of anthropogenic distrurbance along productivity gradient. Forest Ecol. Manag. 250(1):34-46. doi:10.1016/j.foreco.2007.03.007.

Pacheco F. M. 2011. Tabla de volumen para Quercus laurina en la comunidad de Ixtlán de Juárez, Oaxaca. Tesis de Licenciatura. Universidad de la Sierra Juárez. Ixtlán de Juárez, Oaxaca. $63 \mathrm{p}$.

Petritan A. M.; Biris, I. A.; Merce, O.; Turcu, D. O. and Petritan, I. C. 2012. Structure and diversity of a natural temperate sessile oak (Quercus petraea L.)- European Beech (Fagus sylvatica L.) forest. Forest Ecol. Manag. 280:140-149. http://dx.doi.org/10.1016/j.foreco.2012.06.007/.

PROBOSQUE. 1990. Segundo estudio dasonómico del Estado de México (SEDEMEX). Toluca, Estado de México. 334 p.

PROBOSQUE. 2010. Inventario forestal 2010. Toluca, Estado de México. 93 p.

Quintana, R. W. A. 1999. Elaboración de tablas de volumen para aliso (Alnus jorullensis spp. jorullensis Furlow) dentro de la zona de vida bosque muy húmedo montano bajo subtropical en el departamento de Chimaltenango, Guatemala. Tesis de Licenciatura. Universidad de San Carlos de Guatemala, Guatemala. 73 p.

Rodríguez, T. D. y Padilla, G. H. 1976. Tablas de volúmenes para pino (Pinus sp.) y Oyamel (Abies religiosa Schl. et Cham.) del Campo Experimental Zoquiapán. Boletín del Departamento de enseñanza, investigación y servicio en bosques. Información técnica de bosques. Universidad Autónoma Chapingo (UACH). Chapingo, Estado de México. 28-35 pp.

Solís, R.; Aguirre, C. O.; Treviño, G. E.; Jiménez, J.; Jurado, E. y Corral, J. 2006. Efecto de dos tratamientos silvícolas en la estructura de ecosistemas forestales en Durango, México. Madera y Bosques. 12(2):49-64.

Torres, E. L. M.; Sánchez, S. J. A. y Jiménez, P. J. 2006. Análisis estructural de un ecosistema forestal de Pinus-Quercus en la sierra madre oriental. Rev. Cienc. Forest. México. 31(100):7-30.

Vargas-Larreta, B.; Corral-Rivas, J.; Aguirre-Calderón O. y Nagel, J. 2010. Modelos de crecimiento de árbol individual: Aplicación del Simulador BWINPro7. Rev. Madera y Bosques. 16(4):81-104.

Wehenkel C.; Corral-Rivas, J. J.; Hernández-Díaz, J. C. and Gadow, K. 2011. Estimating balanced structure areas in multi-species forests on the Sierra Madre Occidental, México. Ann. Forest Sci. 68:385-394. Doi 10.1007/s13595-011-0027-9.

Zepeda, B. E. M. y Acosta, M. M. 2000. Incremento y rendimiento maderable de Pinus montezumae Lamb, en San Juan Tetla, Puebla. Madera y Bosques. 6(1):15-27.

Zar, J. H. 2010. Biostatistical analysis. Prentice Hall, New Jersey. $5^{\text {th }}$ Edition. 663 p. 